\title{
Análise radiográfica da coluna cervical em indivíduos assintomáticos submetidos a tração manual*
}

\author{
Radiographic analysis of the cervical spine in healthy individuals submitted to manual traction
}

\author{
Roger Burgo de Souza ${ }^{1}$, Edson Lopes Lavado ${ }^{2}$, Fausto Orsi Medola ${ }^{3}$, Dirceu Henrique Blanco ${ }^{4}$, \\ João Henrique Blanco ${ }^{5}$
}

Resumo OBJETIVO: Avaliar, radiograficamente, o efeito da tração manual sobre o comprimento da coluna cervical. MATERIAIS E MÉTODOS: Cinqüenta e cinco participantes de ambos os gêneros - 12 masculinos (22\%) e 43 femininos (78\%) - sem história de distúrbios cervicais contituíram a amostra deste estudo. Eles foram submetidos a dois procedimentos radiológicos, um antes e outro durante a tração manual sustentada por 120 segundos. As distâncias entre as bordas anteriores e posteriores da segunda à sétima vértebras cervicais foram mensuradas e comparadas antes e durante a tração manual. RESULTADOS: A mediana da distância anterior antes da tração foi de $8,40 \mathrm{~cm}$ e durante a tração aumentou para $8,50 \mathrm{~cm}(p=0,002)$. A mediana da distância posterior antes da tração foi de $8,35 \mathrm{~cm}$ e durante a tração aumentou para $8,50 \mathrm{~cm}$ $(p<0,001)$. CONCLUSÃO: Os resultados demonstraram que a aplicação da tração manual promoveu aumento estatisticamente significante do comprimento da coluna cervical em indivíduos assintomáticos.

Unitermos: Radiografia; Manipulação da coluna cervical; Exercício de alongamento muscular.

Abstract OBJECTIVE: To evaluate radiographically the effect of manual traction on the length of the cervical spine in
healthy individuals. MATERIALS AND METHODS: The sample of the present study included 55 individuals
-12 men $(22 \%)$ and 43 women $(78 \%)-$ with no previous history of cervical disorders, submitted to two
radiological procedures previously and during manual traction sustained for 120 seconds. Distances between
the anterior and posterior edges from the second to the seventh cervical vertebrae were measured and
compared before and during manual traction. RESULTS: The median of pre-traction anterior length was
$8.40 \mathrm{~cm}$, increasing to $8.50 \mathrm{~cm}$ during the traction $(p=0.002)$; and the median of pre-traction posterior
length was $8.35 \mathrm{~cm}$, increasing to $8.50 \mathrm{~cm}$ during traction $(p<0.001)$. CONCLUSION: Application of
manual traction resulted in a statistically significant increase in the length the cervical spine in healthy
individuals. Keywords: Radiography; Cervical spine manipulation; Muscle stretching exercise.

Souza RB, Lavado EL, Medola FO, Blanco DH, Blanco JH. Análise radiográfica da coluna cervical em indivíduos assintomáticos submetidos a tração manual. Radiol Bras. 2008;41(4):245-249.

\section{INTRODUÇÃO}

A coluna cervical é composta por sete vértebras formando uma ponte de ligação entre a cabeça e o tronco, com a função de sustentar o crânio, manter o alinhamento e

* Trabalho realizado na Universidade Estadual de Londrina (UEL), Londrina, PR, Brasil.

1. Mestre, Docente da Universidade Estadual de Londrina (UEL), Londrina, PR, Brasil.

2. Doutor, Docente da Universidade Estadual de Londrina (UEL), Londrina, PR, Brasil.

3. Especialista em Fisioterapia Neurofuncional, Docente da Universidade Estadual de Londrina (UEL), Londrina, PR, Brasil.

4. Especialista em Diagnóstico por Imagem, Docente da Universidade Estadual de Londrina (UEL), Londrina, PR, Brasil.

5. Fisioterapeuta, Universidade Estadual de Londrina (UEL), Londrina, PR, Brasil.

Endereço para correspondência: Dr. Roger Burgo de Souza. Rua Aristóteles de Oliveira Chueire, 116, Jardim Aragarça. Londrina, PR, Brasil, 86038-540. E-mail: roger.souza@unopar.br; spine@uel.br.

Recebido para publicação em 20/9/2007. Aceito, após revisão, em 14/12/2007. permitir a movimentação da cabeça ${ }^{(\mathbf{1})}$. Este pilar de ossos móveis sobrepostos é sustentado por estruturas ligamentares, constituindo um sistema passivo de estabilidade $^{(2)}$. A dinâmica dessa região fica sob controle de grupos musculares cervicais e, conjuntamente a este conglomerado de estruturas osteomioligamentares, há tecidos cartilaginosos, nervosos, glandulares e componentes arteriovenosos ${ }^{(3)}$.

A terapia manual inclui manipulação, mobilização passiva, terapia de mobilização neuromuscular, tração manual, massagem nos tecidos moles ${ }^{(\mathbf{4 , 5})}$, sendo utilizada nas condutas fisioterápicas como complemento aos procedimentos para a diminuição dos processos álgicos e das alterações osteomioneuroarticulares das regiões cervicais e lombares. A técnica de tração manual cervical consiste na administração de uma força aplicada pelas mãos do fisioterapeuta, com a finalidade de separar os segmentos vertebrais ${ }^{(6)}$. É considerada modalidade terapêutica passível de ser administrada com o paciente nas posições supina ou sentada ${ }^{(7,8)}$.

Estudos biomecânicos têm tentado determinar o mecanismo usado na técnica de tração cervical, incluindo movimentação vertebral, separação do forame intervertebral, melhor ângulo de tração, emprego adequado da força, tempo ideal de tração e deformação ligamentar ${ }^{(5,8)}$. Embora a tração cervical seja amplamente utilizada no tratamento de vários tipos de desordens cervicais compressivas e tensionais, as opiniões são divergentes quanto ao método de aplicação e dos resultados clínicos ${ }^{(9)}$, pois em vários estudos não é demonstrada a relação entre o tempo de aplicação e a 
magnitude da carga, sendo que os tratamentos conservadores permanecem sem padronização ${ }^{(\mathbf{1 0})}$. No entanto, as vantagens da tração manual incluem o fácil posicionamento das mãos, o feedback sensorial, a especificidade da técnica e o conforto do paciente, mantendo este em repouso. Alguns efeitos fisiológicos da tração incluem a descompressão das estruturas articulares, neurológicas e vasculares, o alongamento dos tecidos moles e a estimulação dos mecanorreceptores, para proporcionar alívio da dor e redução do tônus muscular ${ }^{(11)}$.

Há uma variabilidade de aplicação das técnicas de tração cervical, variando entre a tração manual cervical e outros dois tipos de tração cervical mecânica — intermitente e estática - , o que dificulta uma comparação entre os estudos ${ }^{(12)}$. Além disso, são escassos os trabalhos ou procedimentos publicados que mensurem o comprimento da coluna cervical quando submetida a tração cervical manual. A avaliação radiológica é considerada o "padrão ouro", sendo capaz de permitir, radiograficamente, o exame dos limites angulares e lineares dos movimentos da coluna cervical e toda a sua morfologia ${ }^{(\mathbf{1 3})}$. Alguns pesquisadores têm usado o exame radiológico da região cervical para mensurar o ângulo, a separação vertebral e a movimentação por meio de dispositivos mecânicos ${ }^{(\mathbf{1 4 , 1 5})}$.

Este estudo tem o objetivo de avaliar, radiograficamente, em indivíduos assintomáticos, se a aplicação da tração manual promove mudanças no comprimento da coluna vertebral cervical entre a segunda (C2) e sétima (C7) vértebras.

\section{MATERIAIS E MÉTODOS}

Os indivíduos foram convidados a participar do estudo, compondo uma amostra de conveniência, com 55 participantes de ambos os gêneros — 12 masculinos e 43 femininos - com idade entre 19 e 25 anos. Os sujeitos com história pregressa de afecções cervicais, traumatismos recentes ou antigos, alterações posturais, suspeitas gestacionais e uso de medicamentos miorrelaxantes não foram incluídos.

Todos os participantes receberam um formulário de consentimento, o qual explicava os procedimentos a serem adotados durante o experimento, bem como todas as etapas da pesquisa, tendo concordado e assinado termo de consentimento livre e esclarecido aprovado pelo comitê de ética da Instituição, conforme resolução no 196/96 do Conselho Nacional de Saúde/Ministério da Saúde.

\section{Procedimento experimental}

Todos os participantes foram avaliados para coleta de dados sobre: idade, gênero, estatura, massa corpórea. Antes dos procedimentos, todos se familiarizaram com a tração manual cervical, para reduzir erros no posicionamento e na execução da técnica. Eles foram agendados em horários pré-estabelecidos no período matutino para a execução do exame radiológico e da tração manual.

Os filmes de raios- $\mathrm{X}$ foram identificados por números aleatórios previamente estabelecidos por uma tabela randômica, para evitar qualquer identificação na seqüência desses filmes, em antes e durante a tração manual cervical.

Para o exame radiográfico adotou-se postura inicial em decúbito dorsal sobre a maca do aparelho de raios-X modelo DC15KB, 500 mA (Toshiba; Tóquio, Japão). Todos foram orientados a deixar a região cervical desnuda e sem qualquer adereço metálico que interferisse na execução da técnica. A coluna cervical foi posicionada com o mento a $90^{\circ}$ em relação à superfície da mesa, confirmada por meio de um goniômetro plástico modelo grande, em plástico transparente com duas réguas de mensuração e transferidor de $0^{\circ}$ a $360^{\circ}$ (Carci; São Paulo, Brasil).
Cada participante foi instruído a deprimir os ombros, para permitir adequada visualização do pescoço, e a manter o corpo em postura estática e relaxada, após a confirmação da posição pelo goniômetro, até que terminasse todo o procedimento radiológico e da tração manual cervical. Na seqüência, o fisioterapeuta posicionou-se na cabeceira da mesa. Com sua mão direita, fixou o corpo da mandíbula do participante, e a mão esquerda ficou posicionada sob a região occipital, com o primeiro e o terceiro dedos em contato com o processo mastóide de cada lado do crânio do indivíduo. Em seguida, o técnico em radiologia posicionou o canhão de feixe de incidência dos raios-X, calibrado em $50 \mathrm{kV}$ de penetração e 0,8 mAs de tempo de radiação, direcionado para o centro da quarta vértebra cervical, na lateral do pescoço, a uma distância de $80 \mathrm{~cm}$, obtendo filme radiográfico simples na incidência em perfil (radiografia inicial), em tamanho padronizado de $24 \mathrm{~cm} \times 30 \mathrm{~cm}$. Após a confirmação, pelo técnico em radiologia, de que o filme radiográfico estava em adequadas condições para a visualização de todas as vértebras cervicais, o terapeuta iniciou a aplicação da tração cervical manual. Para isto, foi aplicada uma força de separação no sentido longitudinal entre a cabeça e o tronco, e mantida por 120 segundos. Foi então efetuado outro disparo do canhão de feixe de raios-X, para obtenção do segundo filme radiográfico (radiografia final) (Figura 1). No caso de falha na revelação do filme radiográfico, falha do equipamento ou mudança da postura, os indivíduos foram

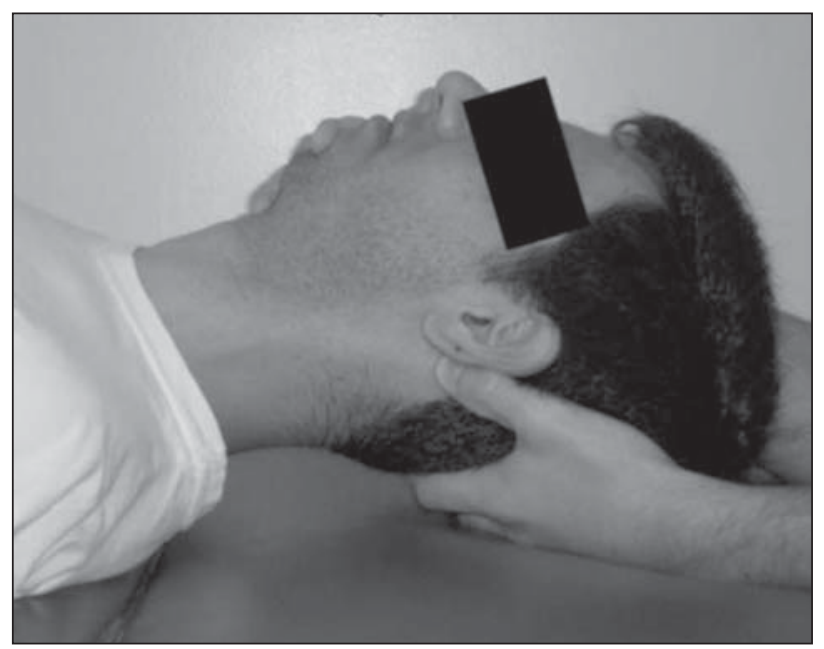

Figura 1. Posicionamento do participante e das mãos do fisioterapeuta para a realização dos procedimentos radiológicos antes e durante a tração manual. 
agendados para repetição do procedimento após trinta dias.

\section{Procedimento para a mensuração dos filmes radiográficos}

Cento e dez filmes radiográficos, antes e durante a tração manual cervical, foram numerados ( 0 a 110) de modo aleatório por meio de uma tabela randômica, para que o avaliador estivesse cego nas mensurações. Os filmes foram colocados em um envelope devidamente identificado com o nome do projeto de pesquisa, o qual foi enviado ao médico radiologista para a emissão das mensurações das distâncias entre C2 e C7. Para a obtenção dessas medidas, o avaliador usou o método de Gore ${ }^{(16)}$, que consiste em traçar uma linha tangenciando a borda inferior do corpo vertebral de C2 e outra sobre a borda superior do corpo vertebral de C7. Após serem traçadas estas linhas tangenciais sobre as radiografias simples de incidência em perfil, mediram-se as distâncias entre os vértices anteriores e entre os vértices posteriores das respectivas vértebras, em centímetros (Figura 2).

Em todas as fases do presente trabalho, o fisioterapeuta, o técnico de radiologia e o médico radiologista foram os mesmos, para evitar qualquer viés nos procedimentos realizados.

\section{Análise estatística}

A estatística descritiva expressou os valores da média e desvio-padrão ou me-

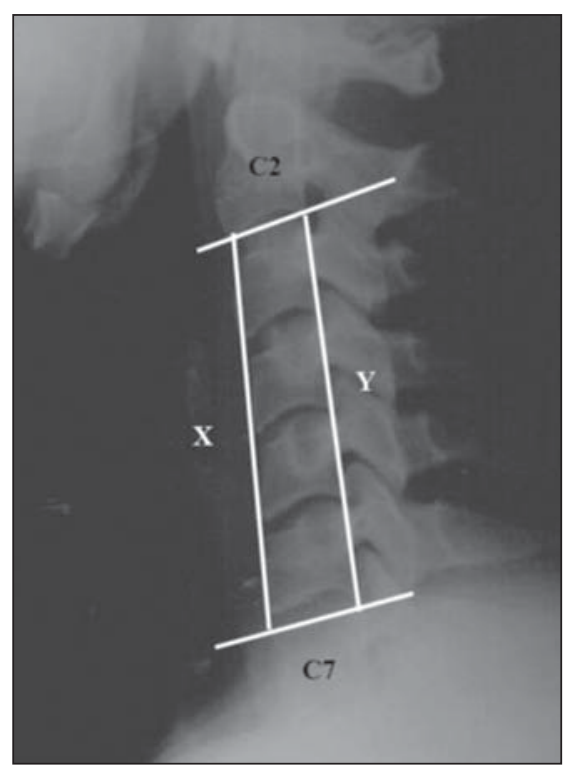

Figura 2. Mensuração do comprimento cervical pelo método de Gore. $X$, distância entre as bordas anteriores; $Y$, distância entre as bordas posteriores de $\mathrm{C} 2$ a $\mathrm{C} 7$.

diana, quartis e valores mínimos e máximos, apresentados por figuras e tabelas. Para a comparação das variáveis idade, massa corpórea, estatura e índice de massa corporal, utilizou-se o teste $t$ de Student. $\mathrm{Na}$ comparação dos achados antes e durante a tração manual cervical, foi utilizado o teste de Wilcoxon. Utilizou-se o programa Statistical Package for Social Sciences, versão 13.0. Para todos os testes mencionados, a significância estatística foi estabelecida em $5 \%(p=0,05)$.

\section{RESULTADOS}

Entre os 55 indivíduos participantes do estudo, 12 (22\%) eram do gênero masculino e 43 (78\%) eram do gênero feminino. Foram registradas as seguintes médias: de idade $=21,20 \pm 1,40$ anos; da massa corpórea $=58,40 \pm 9,70 \mathrm{~kg}$; da estatura $=1,66$ $\pm 0,08 \mathrm{~m}$; e do índice de massa corporal = $20,96 \pm 2,14 \mathrm{~kg} / \mathrm{m}^{2}$.

Em relação ao comprimento da coluna cervical, na comparação das medidas entre as bordas vertebrais anteriores antes da tração, a mediana foi de $8,40 \mathrm{~cm}$ (mínima = $7,05 \mathrm{~cm}$; máxima $=10,50 \mathrm{~cm} ; 1^{\circ}$ quartil $=$ $8,00 \mathrm{~cm} ; 3^{\circ}$ quartil $\left.=9,00 \mathrm{~cm}\right)$, e durante a tração foi de $8,50 \mathrm{~cm}$ (mínima $=7,20 \mathrm{~cm}$; máxima $=10,90 \mathrm{~cm} ; 1^{\circ}$ quartil $=8,20 \mathrm{~cm}$; $3^{\circ}$ quartil =9,20 cm). Quando foram comparados os valores das bordas anteriores antes e durante a tração, observou-se diferença estatisticamente significante ( $p=$ 0,002) (Figura 3).

Na comparação das medidas entre as bordas vertebrais posteriores antes da tração, a mediana foi de $8,35 \mathrm{~cm}$ (mínima = $7,10 \mathrm{~cm}$; máxima $=10,30 \mathrm{~cm} ; 1^{\circ}$ quartil $=$ $7,95 \mathrm{~cm} ; 3^{\circ}$ quartil $\left.=8,70 \mathrm{~cm}\right)$, e durante a tração foi de $8,50 \mathrm{~cm}$ (mínima $=7,30 \mathrm{~cm}$; máxima $=10,50 \mathrm{~cm} ; 1^{\circ}$ quartil $=8,00 \mathrm{~cm}$; $3^{\circ}$ quartil $=8,90 \mathrm{~cm}$ ). Quando foram comparados os valores das bordas posteriores antes e durante a tração, observou-se diferença estatisticamente significante ( $p<$ 0,001) (Figura 4).

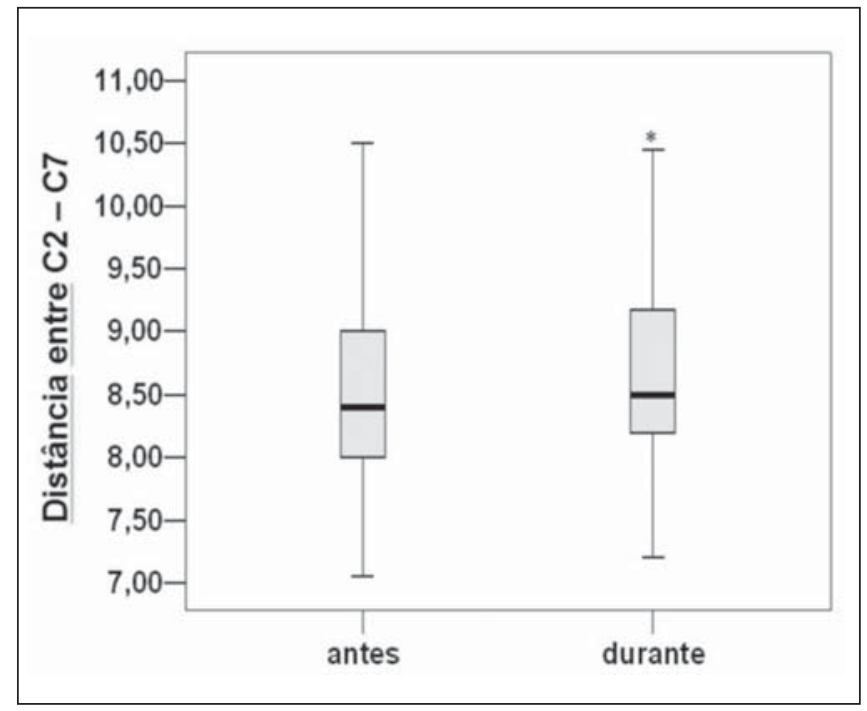

Figura 3. Distância entre as bordas vertebrais anteriores antes e durante a tração cervical manual. * Houve diferença estatísticamente significante.

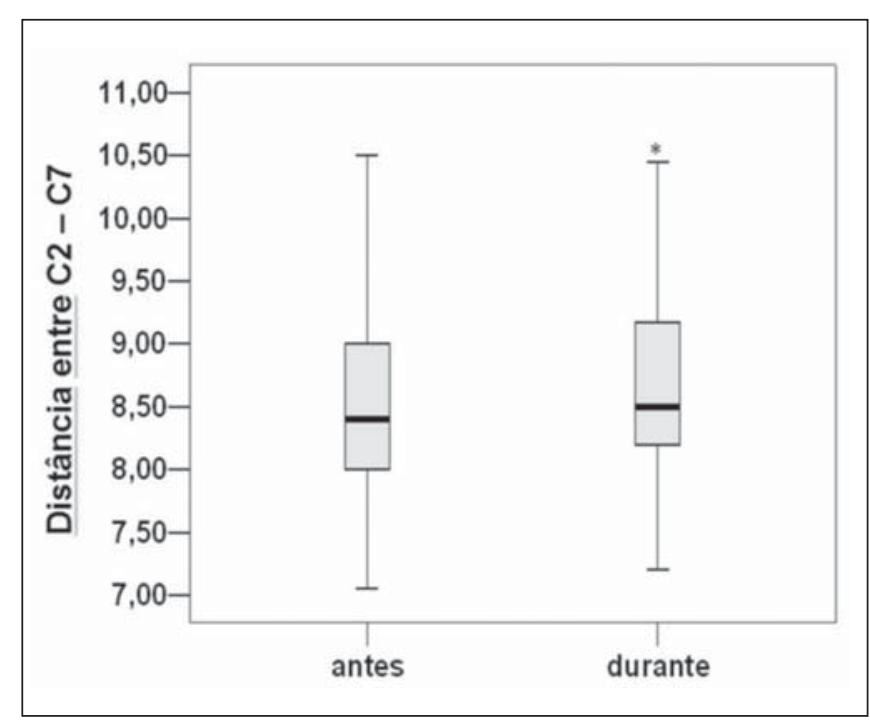

Figura 4. Distância entre as bordas vertebrais posteriores antes e durante a tração cervical manual. * Houve diferença estatisticamente significante. 


\section{DISCUSSÃO}

A mensuração do comprimento da coluna cervical por meio da radiografia mostrou ser procedimento simples, de baixo custo e de fácil execução. A escolha do procedimento radiológico foi pautada pelo custo-benefício para esta pesquisa, pois a avaliação radiológica é considerada o "padrão ouro", sendo capaz de permitir, radiograficamente, o exame dos limites angulares e lineares dos movimentos da coluna cervical e de toda a sua morfometria ${ }^{(\mathbf{1 3 , 1 7})}$. Embora a ressonância magnética seja o melhor método para demonstrar as condições e diferenças teciduais, ela ainda é de alto custo, consome tempo e muitas vezes não está disponível em nosso meio ${ }^{(18)}$.

Em estudo de Vaughn et al. ${ }^{(14)}$, a análise radiográfica em indivíduos assintomáticos foi conduzida para investigar o ângulo e o aumento do espaço intervertebral com tração mecânica ou com cargas pré-estabelecidas. No presente trabalho, os resultados mostraram que a força empregada pelo fisioterapeuta aumentou o comprimento da coluna cervical entre $\mathrm{C} 2$ e $\mathrm{C} 7$ em indivíduos assintomáticos, mensurado por meio das radiografias simples em perfil. Podese concluir que a tração manual promove aumento dos espaços intervertebrais e relaxamento das estruturas musculares, como nos resultados de estudos anteriores com utilização de tração mecânica ${ }^{(\mathbf{8}, 17,19)}$.

Embora a tração cervical seja amplamente usada no tratamento de vários tipos de desordens cervicais compressivas e tensionais, as opiniões são divergentes quanto ao método de aplicação e resultados clíni$\cos ^{\left({ }^{(9)}\right.}$, pois em vários estudos não é demonstrada a relação entre o tempo de aplicação e a magnitude da carga, e os tratamentos conservadores permanecem sem padronização ${ }^{(10)}$. No presente estudo, houve a preocupação quanto ao posicionamento do participante em supino, para eliminar qualquer postura inadequada, à manutenção da coluna cervical em posição neutra, permitindo um único vetor de força, e ao tempo (120 segundos), previamente estabelecido conforme o período que o terapeuta conseguiu sustentar a força de tração.

Entende-se que esta técnica de tração é empregada com o intuito de promover os alongamentos musculares da coluna cervi- cal, bem como o aumento dos espaços intervertebrais, os quais, tensionados e diminuídos, respectivamente, são as etiologias dos processos álgicos e compressivos desta região ${ }^{(7)}$. O efeito mecânico da tração cervical diminui o déficit de preensão palmar de pacientes com radiculopatias cervicais $^{\left({ }^{(8)}\right.}$. As vantagens da tração manual em relação à tração mecânica incluem o fácil posicionamento das mãos, o feedback sensorial do toque, a especificidade da técnica e o conforto do paciente, mantendo-o em repouso. Alguns efeitos fisiológicos da tração incluem a descompressão das estruturas articulares, neurológicas e vasculares, o alongamento dos tecidos moles e a estimulação dos mecanorreceptores, com a finalidade de proporcionar alívio da dor e redução do tônus muscular ${ }^{(11)}$. Entretanto, deve-se ter o conhecimento do diagnóstico médico e realizar uma avaliação cinesiológica funcional, com o intuito de programar o tratamento adequado para os comprometimentos cervicais.

Em outro estudo, pacientes com diagnóstico de radiculopatia com herniação maior que $4 \mathrm{~mm}$ foram tratados com tração cervical intermitente e avaliados por meio de ressonância magnética. A tração era mantida por 45 minutos durante seis a oito horas com cargas pré-estabelecidas. Todos os pacientes tiveram melhora dos sintomas e diminuição da hérnia discal ${ }^{(\mathbf{1 9})}$. Ainda que no presente trabalho não tenham sido mensuradas as distâncias entre cada corpo vertebral, pode-se concluir que a força longitudinal aplicada favorece a diminuição da pressão entre os corpos vertebrais, propiciando um aumento dos espaços intervertebrais, aumentando, assim, o comprimento da coluna cervical.

Chung et al. ${ }^{(17)}$ estudaram um grupo assintomático e outro com diagnóstico de protrusão discal cervical, nos quais aplicaram tração cervical intermitente durante as atividades de vida diária, utilizando um dispositivo pneumático calibrado em 30 libras de força. Na seqüência, submeteram os indivíduos a exame por ressonância magnética, que demonstrou aumento no comprimento da coluna cervical. No grupo assintomático houve aumento de $1,93 \mathrm{~mm}$ e no grupo com hérnia, de 2,19 mm, ocorrendo significativa redução da protrusão discal. Esses resultados foram similares aos nos- sos achados, confirmando, mais uma vez, que a tração cervical altera o comprimento da região cervical, e quando aplicada manualmente, produz alterações equivalentes à tração mecânica.

Em trabalho com colunas cervicais de cadáveres tracionadas mecanicamente e associadas a flexão da coluna cervical, Humphreys et al. observaram aumento de $3 \mathrm{~mm}^{2}$ a $4 \mathrm{~mm}^{2}$ da área do forame intervertebral $^{(20)}$. Porém, esses resultados não podem ser equivalentes aos de indivíduos vivos. No presente trabalho, não foi investigado o forame, mas acredita-se que o somatório desses valores resultaria em aumento do comprimento longitudinal da coluna cervical em indivíduos vivos.

Ao contrário dos resultados do presente trabalho, Harrison et al. ${ }^{(5)}$ encontraram diminuição do comprimento entre as bordas posteriores dos corpos vertebrais de $\mathrm{C} 2 \mathrm{a}$ $\mathrm{C} 7$, de aproximadamente $5 \mathrm{~mm}$ durante a tração associada à extensão mecânica da região cervical. Considera-se que esta associação promoveu o aumento da lordose cervical, resultando na redução do comprimento posterior dos corpos vertebrais cervicais. No presente trabalho houve a preocupação com o posicionamento para manter a coluna cervical na posição neutra, evitando compensações da curvatura cervical. Há comprovação de que na extensão da coluna cervical ocorre um deslizamento facetário entre as vértebras cervicais, contribuindo para uma aproximação posterior dos corpos vertebrais ${ }^{(1)}$.

Durante todas as etapas do presente trabalho, nenhum participante referiu desconforto ou dor durante ou posteriormente à tração manual cervical. Apesar de a técnica de tração manual cervical conseguir promover uma alteração na coluna cervical, a qual se acredita ser benéfica, os autores do presente estudo depararam-se com a limitação sobre o grau de força manual que deve ser aplicada durante a tração para se obter o aumento dos espaços intervertebrais, uma vez que em estudos prévios todos os procedimentos de tração cervical eram com cargas pré-definidas ${ }^{(\mathbf{8 , 2 0})}$. Os autores acreditam que outra limitação foi a de ter a amostra de conveniência formada por indivíduos assintomáticos, o que não permitiu avaliar os sintomas que poderiam aparecer ou diminuir antes, durante ou após 
a tração manual. E uma outra, foi a média das idades, de 19 a 25 anos, que não representa adequadamente a população adulta com disfunções cervicais.

No presente estudo ficou demonstrado que a tração manual cervical aumenta o comprimento da coluna cervical, promovendo maior distanciamento entre os corpos vertebrais, descompressão radicular e relaxamento muscular cervical, devendo ser um complemento dos procedimentos terapêuticos incorporados em um programa de fisioterapia.

\section{CONCLUSÃO}

Os resultados foram satisfatórios quando as medidas da coluna cervical foram comparadas antes e durante a tração manual cervical, demonstrando que a tração produziu aumento estatisticamente significante do comprimento da coluna cervical. A aplicação de exercícios terapêuticos específicos, como a tração cervical manual, deve ser o diferencial na obtenção de um prognóstico melhor para os comprometimentos osteomioneuroarticulares da coluna cervical. Portanto, a análise radiológica irá auxiliar na interpretação e constatação dos benefícios da intervenção fisioterapêutica. No entanto, quanto à sua aplicação em indivíduos sintomáticos e adequação do comprimento cervical, bem como a mensuração da força que deve ser aplicada manualmente nesses procedimentos, faz-se necessária a aplicação de estudos clínicos aleatórios para subsídios de uma aplicação terapêutica eficaz.

\section{Agradecimentos}

À direção do Setor de Radiologia do Hospital Universitário, a Jaime Stelli, técnico em radiologia, pela disposição e agendamento dos procedimentos radiológicos, e à direção clínica, na pessoa do Dr. Sinésio Moreira Junior, pela autorização e acompanhamento na execução deste projeto, bem como a todos os participantes, nossos sinceros agradecimentos.

\section{REFERÊNCIAS}

1. Mercer SR, Bogduk N. Joints of the cervical vertebral column. J Orthop Sports Phys Ther. 2001; 31:174-82.

2. Olson KA, Joder D. Diagnosis and treatment of cervical spine clinical instability. J Orthop Sports Phys Ther. 2001;31:194-206.

3. Nordin M, Frankel VH. Biomecânica da coluna cervical. In: Nordin M, Frankel VH, editores. Biomecânica básica do sistema musculoesquelético. $3^{\mathrm{a}}$ ed. Rio de Janeiro: Guanabara Koogan; 2003. p. 250-60.

4. Gross AR, Kay T, Hondras M, et al. Manual therapy for mechanical neck disorders: a systematic review. Man Ther. 2002;7:131-49.

5. Harrison DE, Cailliet R, Harrison DD, et al. A new 3-point bending traction method for restoring cervical lordosis and cervical manipulation: a nonrandomized clinical controlled trial. Arch Phys Med Rehabil. 2002;83:447-53.

6. Carette S, Fehlings MG. Cervical radiculopathy. N Engl J Med. 2005;353:392-9.

7. Nadler SF. Nonpharmacologic management of pain. J Am Osteopath Assoc. 2004;104(11 Suppl 8):S7-12.

8. Joghataei MT, Arab AM, Khaksar H. The effect of cervical traction combined with conventional therapy on grip strength on patients with cervical radiculopathy. Clin Rehabil. 2004;18:879-87.

9. Wong AMK, Lee MY, Chang WH, et al. Clinical trial of a cervical traction modality with electromyographic biofeedback. Am J Phys Med Rehabil. 1997;76:19-25.
10. Rodacki ALF, Weidle CM, Fowler NE, et al. Changes in stature during and after spinal traction in young male subjects. Rev Bras Fisioter. 2007;11:63-71.

11. Makofsky HW. Técnicas para os tecidos conjuntivos e procedimentos de alongamentos para a coluna cervical. In: Makofsky HW, editor. Coluna vertebral: terapia manual. $1^{\text {a }}$ ed. Rio de Janeiro: Guanabara Koogan; 2006. p. 90-101.

12. Peake N, Harte A. The effectiveness of cervical traction. Physical Therapy Reviews. 2005; 10: 217-29.

13. Wolfenberger VA, Bui Q, Batenchuk GB. A comparison of methods of evaluating cervical range of motion. J Manipulative Physiol Ther. 2002;25: 154-60.

14. Vaughn HT, Having KM, Rogers JL. Radiographic analysis of intervertebral separation with a $0^{\circ}$ and $30^{\circ}$ rope angle using the Saunders cervical traction device. Spine. 2006;31:39-43.

15. Fernández-de-las-Peñas $\mathrm{C}$, Downey C, Miangolarra-Page JC. Immediate changes in radiographically determined lateral flexion range of motion following a single cervical HVLA manipulation in patients presenting with mechanical neck pain a case series. Int J Osteopath Med. 2005;8:13945.

16. Rowe LJ, Yochum TR. Measurements in skeletal radiology. In: Rowe LJ, Yochum TR, editors. Essential of skeletal radiology. 2nd ed. New York: Williams \& Wilkins; 1999. p. 153-4.

17. Chung TS, Lee YJ, Kang SW, et al. Reducibility of cervical disk herniation: evaluation at MR imaging during cervical traction with a nonmagnetic traction device. Radiology. 2002;225:895-900.

18. Nucci CG, Caiero MT, Lasmar RCP, et al. Estudo radiográfico da coluna cervical em crianças com artrite reumatóide juvenil. Rev Bras Ortop. 1999; 34:117-27

19. Constantoyannis C, Konstantinou D, Kourtopoulos $\mathrm{H}$, et al. Intermittent cervical traction for cervical radiculopathy caused by large-volume herniated disks. J Manipulative Physiol Ther. 2002;25:188-92

20. Humphreys SC, Chase J, Patwardhan A, et al. Flexion and traction effect on C5-C6 foraminal space. Arch Phys Med Rehabil. 1998;79:1105-9. 